\title{
Lights and Shadows of the Perception of the Use of Telemedicine by Romanian Family Doctors During the COVID-19 Pandemic
}

\author{
Mira Florea $\mathbb{D}^{1}$ \\ Cecilia Lazea ${ }^{2}$ \\ Remus Gaga (iD) ${ }^{2}$ \\ Genel Sur ${ }^{2}$ \\ Lucia Lotrean' \\ Aida Puia' \\ Ana Maria Alexandra Stanescu ${ }^{3}$ \\ Monica Lupsor-Platon ${ }^{4}$ \\ Horatiu Florea $^{5}$ \\ Maria Lucia Sur ${ }^{2}$ \\ 'Department of Community Medicine, \\ "Iuliu Hatieganu" University of Medicine \\ and Pharmacy, Cluj-Napoca, Romania; \\ ${ }^{2}$ Department of Pediatrics, "Iuliu \\ Hatieganu" University of Medicine and \\ Pharmacy, Cluj-Napoca, Romania; \\ ${ }^{3}$ Department of Family Medicine, "carol \\ Davila" University of Medicine and \\ Pharmacy, Bucharest, Romania; ${ }^{4}$ Medical \\ Imaging Department, "Iuliu Hatieganu" \\ University of Medicine and Pharmacy, \\ Cluj-Napoca, Romania; ${ }^{5}$ Computer \\ Science Department, Technical University \\ of Cluj-Napoca, Cluj-Napoca, Romania
}

Correspondence: Cecilia Lazea $\checkmark$ Babes 8 Street, Cluj-Napoca, 400006, Romania

Tel + 40744353764

Email cecilialazea@umfcluj.ro
Introduction: Telemedicine has emerged as a critical technology to mitigate SARS-CoV-2 infection. We aim in this work to explore how general practitioners (GPs) perceived the use of telemedicine, recently recognized and reimbursed by the Public Health Insurance House (PHIH) for primary care (PC) provision.

Methods: A cross-sectional study was performed in 2020 in one county of Romania using an anonymous questionnaire that assessed physicians' perceptions regarding teleconsultation, reliability in tele-decision, remote pathology management, pregnant women's surveillance, patients' satisfaction with telemedicine, the need for its further reimbursement. Bivariate correlation was used to measure associations between the investigated issues.

Results: More than a quarter of GPs $(28.6 \%)$ found it easier to address patients' healthcare needs remotely, while $60.7 \%$ considered time-consuming teleconsultations compared to faceto-face visits. Tele-diagnostic uncertainty was expressed by $64.3 \%$ of physicians, and a quarter were confident in tele-decisions. Almost half of GPs (43\%) observed patients' satisfaction with tele-visits, while half said patients encountered difficulties using technology. A large percentage of doctors $(62.5 \%)$ perceived that patients felt as well treated by virtual as in-person visit and $91.1 \%$ suggested post-pandemic reimbursement. The results of the bivariate correlation showed that physicians who perceived positive patient feedback on telemedicine were more supportive of subsequent reimbursement.

Conclusion: This study showed the GPs' positive perception of the use of telemedicine. Its adoption in PC has shed light on the shadows of the pandemic. The time-consuming nature of teleconsultations, uncertainty in tele-decisions, patients' difficulties in using technology were seen as shadows of telecare. However, most of the GPs surveyed agreed with the need for further reimbursement. Future work should focus on innovative solutions for integrating telemedicine as complementary form of PC, the need for telemedicine-based training for GPs to improve capacity building, and patients' perceptions of virtual care, helping to build trust and satisfaction.

Keywords: telemedicine, primary care, family doctor, perception, Covid-19 pandemic

\section{Plain Language Summary}

- The recognition and reimbursement of telemedicine by public health authorities during the COVID-19 pandemic in Romania brought light into the shadow of the pandemic.

- Our study describes the perception of the use of telemedicine by family doctors during the COVID-19 in lights and shadows.

- We considered as lights: the rapid adjustment of doctors to tele-decisions, their proposal to reimburse telemedicine beyond the pandemic, the positive reaction of 
patients to the critical transition from conventional to virtual consultation, almost two-thirds of them feeling as well treated by virtual as in-person visit and a quarter observing a greater availability of solving their needs through telecare.

- The shadows of the rapid transition to remote alternative care identified by our study were: longer duration of teleconsultations, uncertainty in making remote medical decisions and difficulties of some patients in using communication technology.

- Although telemedicine was initially reimbursed only during the state of emergency, its contribution to pandemic mitigation and positive perception by both healthcare providers and patients led $\mathrm{PHIH}$ to continue reimbursement during the COVID-19 pandemic.

\section{Introduction}

Telemedicine has been rapidly evolving over the past several decades, but it has not yet been widely implemented into the healthcare system due to regulatory laws of concern and lack of supportive payment structures. ${ }^{1,2}$ The term telemedicine was initially used to refer to the provision of remote healthcare services using information and communications technologies (ICT). Telehealth is a term that was introduced as a result of the widespread use of telemedicine, such as medical education and health systems management. New terms like e-health, m-health and connected health have emerged, recently. E-health was designed to refer to a wide range of data processing applications, the use of the internet in health systems and health promotion applications. Telehealth and e-health can be considered as an extension of the original term telemedicine. However, the terms telemedicine, telehealth and e-health are often used interchangeably by both healthcare professionals and consumers. $^{3,4}$ Telemedicine is implemented using ICT either asynchronously or synchronously via audio and video systems. 5,6

Prior to the Coronavirus 2019 pandemic (COVID-19), regulatory and reimbursement issues prevented the full introduction of telemedicine into the health system in many countries. ${ }^{2}$

\section{Literature Review}

There is a growing interest in its adoption to provide healthcare, in its potential to reduce the exposure of patients and healthcare professionals to the risk of SARSCoV-2 infection, to preserve protective equipment, to reduce delays in caring for non-covid patients and to respond to patients' preference for virtual visits due to fear of exposure to infection.

Telecare emerged as an effective solution for prevention and treatment of new coronavirus infection, contributing positively to the safe provision of primary care (PC) during the pandemic. Since 2005, the World Health Organization (WHO) established a global observatory for e-health to monitor the development of ICT for health care, including telemedicine, and to provide reliable guidance on best practices and standards. ${ }^{7}$ It has progressed far less in lower-income countries than in high-income countries both in terms of the number of countries with established services and the proportion of telemedicine services offered. In April 2020, the WHO mentioned telemedicine among its key services in "strengthening the response of health systems to the COVID-19 pandemic". 8

The current pandemic prompted outpatient medical centers to change their health delivery system, to include tele-visits supporting the continuity of care. Telemedicine should be seen not only as a temporary emergency alternative, but as a convenient, safe, scalable and effective way to provide healthcare and increase access to quality care. ${ }^{9}$ In addition to virtual visits, text applications, e-mail and mobile telephony, telemedicine facilitates the exchange of information not only between patients and doctors, but also interprofessional communication between family doctors and specialists. ${ }^{5}$

Over the past several years, researchers have been exploring the advantages and disadvantages of telemedicine compared with face-to-face consultation. The advantages of telemedicine: cost-effectiveness, increased access to specialized services, its potential to help mitigate SARS- CoV-2 infection and alleviate the current or emerging physicians shortage in many countries are well highlighted by this pandemic.

Disadvantages include lack of available technological resources in low-income and some middle-income countries, issues with patient data security, physicians' uncertainty in tele-decisions because of the challenges in conducting patient examination. ${ }^{1}$ While telemedicine is gradually delivered through smart devices, the technology usually requires both the patient and physician to learn how to use these platforms.

Training is needed to help physicians provide remote healthcare, which requires knowledge and upskilling to be able to use virtual technology and equipment. Moreover, patients need to be educated so that they can be aware of virtual healthcare solutions and their benefits. Telemedicine 
could be publicized through social networks to create awareness, as elderly people may have difficulty using ICT. ${ }^{10}$

There are countries where policy changes have focused on improving reimbursement models and the development of digital infrastructure, thus facilitating the acceleration of telemedicine adoption. ${ }^{11}$

Lack of official recognition of teleconsultation before the Covid-19 pandemic in Romania, a member country of the European Union, with high internet speed, but which failed to finance them, although they were provided for years by general practitioners (GPs), especially in rural areas, justifies the need to research how they perceived the adoption of telemedicine during this crisis and how they see the improvement of future health policy.

When the state of emergency was decided at the national level, the public health authorities (PHA) encouraged family doctors to provide healthcare services remotely, as one of the measures taken to increase social distancing and home confinement. Moreover, in response to the needs of the pandemic and the WHO recommendations of April 2020, that "other countries will make their decision in the coming weeks to include telemedicine as a Standard Operating Procedure (SOP) for the prevention and treatment of COVID-19", ${ }^{12}$ the executive decided for the first time in Romania by Government Decision 252/ 30 March 2020, the reimbursement of teleconsultations in PC starting with March 30, 2020.

During the state of emergency, and then in the alert status, GPs provided remote consultations, which could be performed by any means of communication, with a maximum of 8 consultations/hour. Patients received teleconsultations for any symptoms suggestive of coronavirus infection and other pathologies, even if they did not have public health insurance and were informed about the limits of tele-visit and the need to call the GP's office or the emergencies department if symptoms worsen.

There are fewer studies focused on the adoption of telemedicine to support provision of PC. Considering the importance that changes are made to adopt and finance telemedicine services during the pandemic into the Romanian healthcare, a research on GPs' perception of the critical transition to virtual visits and how they saw patients' satisfaction with this approach is justified. As the pandemic continues and evolves, physicians' opinion on the future of telemedicine and how they expect this technology to be implemented after the COVID-19 pandemic are of interest.
We aim in this work to explore how family physicians perceived the use of telemedicine, recently recognized as a tool for PC provision and reimbursed for the first time by the Romanian Public Health Insurance House (PHIH). In addition, we analyzed their opinions on the need for its further reimbursement and patients' satisfaction using ICT as perceived by family doctors.

\section{Materials and Methods Procedure for Data Collection}

In this cross-sectional study, we developed an anonymous questionnaire and distributed it online between April and September 2020 to 108 family doctors in Cluj County, a mountainous region in northwestern Romania, with many isolated rural areas, with 737,992 inhabitants. ClujNapoca is the third city in Romania, being one of the most important academic centers, with 308,000 citizens.

The questionnaire aimed to assess family physicians' views on telemedicine as PC service tool adopted during the emergency state of the Covid-19 pandemic. It was administered online as a Google form in Romanian language and took approximately 13 minutes to complete.

The studied population targeted for this online survey was represented by GPs who practiced in PC offices in Cluj County. The invitation to participate was sent to family doctors from a mailing list established over several years of collaborative projects between the Department of Family Medicine at the University of Medicine and Pharmacy and The Society of Family Doctors from Cluj. The objectives and characteristics of the study were clearly explained, and participation was voluntary by accessing the link. By filling in the questionnaire, the participants agreed to participate; physicians who refused to participate did not complete the questionnaire. The response rate of family doctors during the demanding COVID-19 pandemic, including the state of emergency and part of the alert period was $51 \%$.

\section{Instrument for Data Collection}

The questions of the designed questionnaire were grouped into sections and coded as follows.

\section{Perception of Teleconsultations and Reliability in Remote Decision Making}

Participants were asked to rate the teleconsultation compared to in-person visit, possibilities of answers being: "more difficult and time consuming" (coded as -1 ), "the 
same" (coded as 0), "easier" (coded as 1). Another question investigated their confidence in making decisions remotely, with possible answers: "the correct establishment of the diagnosis and treatment may be affected" $(-1)$,

"I did not notice significant differences" (0), "I consider that telemedicine does not affect the correct establishment of the diagnosis and treatment" (1).

- Perception of the management of acute and chronic pathology and surveillance of pregnant women in primary care during the pandemic

Participants were asked how they perceived the remote management of acute pathology, possibilities of answers being: "I noticed that solving the problem became difficult" (-1), "we could only partially solve the problem" (0) and "as before" (1). Asked how they perceived the chronic pathology management in crisis, the possibilities of answer were: "inadequate, as many sections were intended for COVID-19 patients only" (-1), "were resolved late, by planning to a specialist" (0), "as before" (1)

The question focused on opinion regarding how has been affected the surveillance of pregnant women in PC during the pandemic, was coded: $(-1)$ for "patients have postponed in-person visits in GPs' office",

(0) for "the frequency of checks at the office during the pandemic did not change", (1) for "patients presented to the GPs' office more often than before, preferring inperson consultation.

- Patients'satisfaction with the use of communication technology to address their health care needs in primary care, as perceived by family physicians.

This section has 2 questions about how GPs have noticed that their patients have reacted to telemedicine in terms of technology, respectively the quality of medical services provided, with 3 possibilities to answer: negative reaction $(-1)$, indifference $(0)$ and positive reaction (1). Moreover, they were asked how they think the patients perceived tele-visits, with 3 possible answers: "the patient felt neglected" (-1), "felt equally well treated" (0), "felt better and more promptly treated" (1).

- Opinion of family doctors on the need for further reimbursement of telemedicine by the Public Health Insurance House (PHIH) after the pandemic
The last question on the need to keep teleconsultation as a complementary service and its reimbursement after the pandemic was coded as follows: $(-1)$ for "no, because I do not consider that a teleconsultation has the same accuracy as a classic consultation", (0) for "I do not care" and (1) for "yes, it should be preserved and further reimbursed" (1).

\section{Data Analyses}

The prevalence of the investigated issues was calculated. Three scales were created regarding the perception of teleconsultations and the reliability of remote decisionmaking (Cronbach's alpha $=0.57$ ), the perception of acute and chronic pathology management and the supervision of pregnant women in PC during the pandemic (Cronbach's alpha $=0.62$ ) and patient satisfaction with the use of communication technology to meet their health care needs in PC, as perceived by GPs (Cronbach's alpha $=0.65$ ). Each scale was created by summing the codes of the comprising questions for every participant.

The bivariate correlation was used to assess the correlation between the three scales and the opinion of family doctors on the need to reimburse telemedicine after the pandemic.

Statistical analyses were performed using SPSS 20 statistical program. Results with statistical significance are reported at $\mathrm{p}<0.05$.

\section{Results}

\section{Opinions of Family Doctors Regarding the Use of Telemedicine}

More than a quarter of GPs found it easier to address patients' healthcare needs remotely, while half responded that teleconsultations are time-consuming compared to face-to-face visits (Figure 1.).

Almost two-thirds of the family doctors expressed uncertainty about the correctness of the diagnosis established by tele-visit, and a quarter were confident in making decisions remotely (Figure 2.).

In terms of resolving acute pathology, the perception of half of the family doctors was that they solved it as before, and more than a quarter noticed that the solving became difficult, many of the acute diseases' symptoms being difficult to differentiate from those of the infection with the new coronavirus (Figure 3.).

On the other hand, chronic pathology was more difficult to resolve during this period, with almost half of GPs responding that scheduled reevaluations could not be 
How do you appreciate the telemedicine (teleconsultation) compared to the in person/face to face consultation in the office?

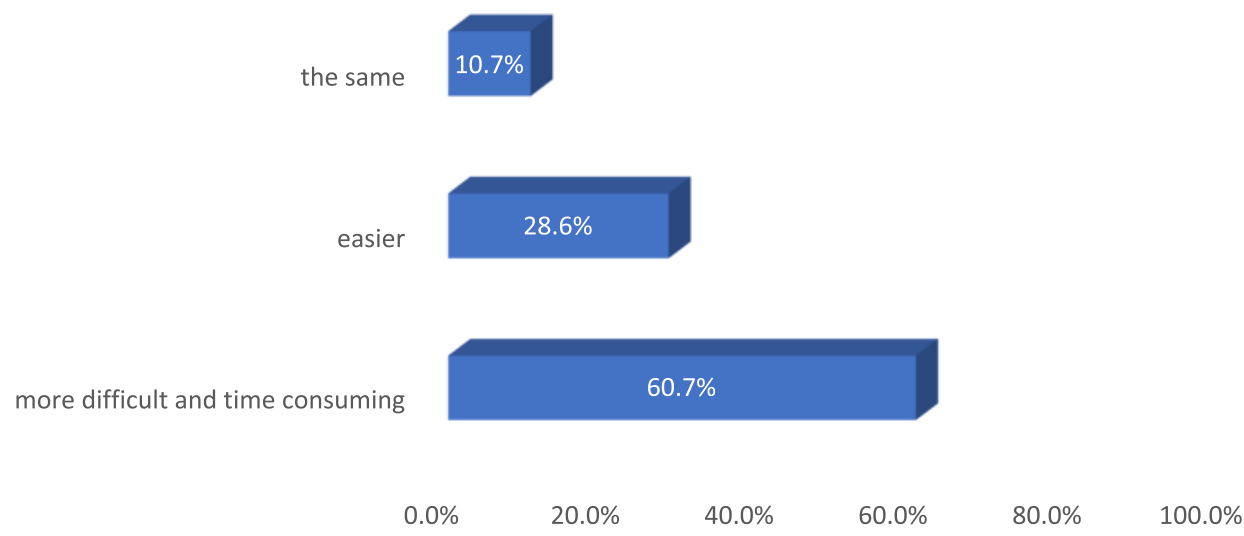

Figure I Perception of teleconsultations by GPs compared to in-person consultations.

How much confidence do you have in making the correct diagnosis through telemedicine?

I did not notice significant differences

I consider that telemedicine does not affect the correct establishment of the diagnosis

the correct establishment of the diagnosis may be affected

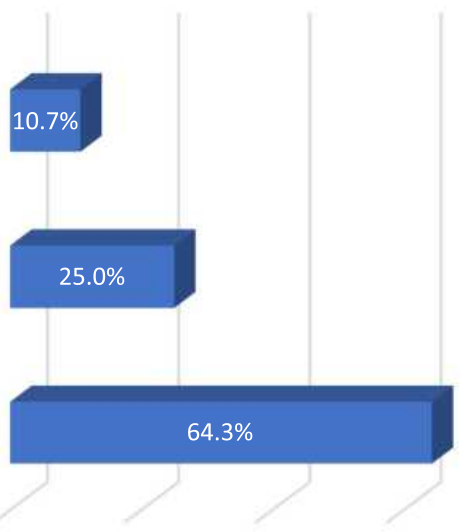

$0.0 \%$

$20.0 \%$

$40.0 \%$

$60.0 \%$

$80.0 \%$

$100.0 \%$

Figure 2 Family doctors' confidence in making decisions remotely.

performed because most hospitals were intended for COVID-19 infections (Figure 4.).

Supervision of pregnant women was affected by the fear of Sars-CoV-2 infection, causing delays in their presentation at scheduled visits. Most family doctors reported that their registered pregnant women preferred teleconsultations and video consultations, while a third said that the frequency of consultations in their offices for pregnancy surveillance has not changed (Figure 5.).

According to the periodic assessment of patient satisfaction by family physicians, almost half reported that their patients were satisfied with this way of interaction and the use of communication technology, which simplified the solution of their healthcare needs (telephone consultations, WhatsApp video consultations, prescription received electronically). The other half of GPs said, on the contrary, that patients encountered difficulties using communication technology with the doctors and nurses (Figure 6.).

In terms of the quality of healthcare services provided, half of family physicians noticed that for their patients the quality of virtual and in-person consultations seemed to be the same. However, a quarter of GPs considered that their patients felt insecure about the accuracy of the diagnosis in the absence of an objective examination, while another quarter appreciated that patients were equally satisfied with teleconsultation as well as in-person visit to the office (Figure 7.). 
How did you perceive the remote management of acute pathology during the pandemic?

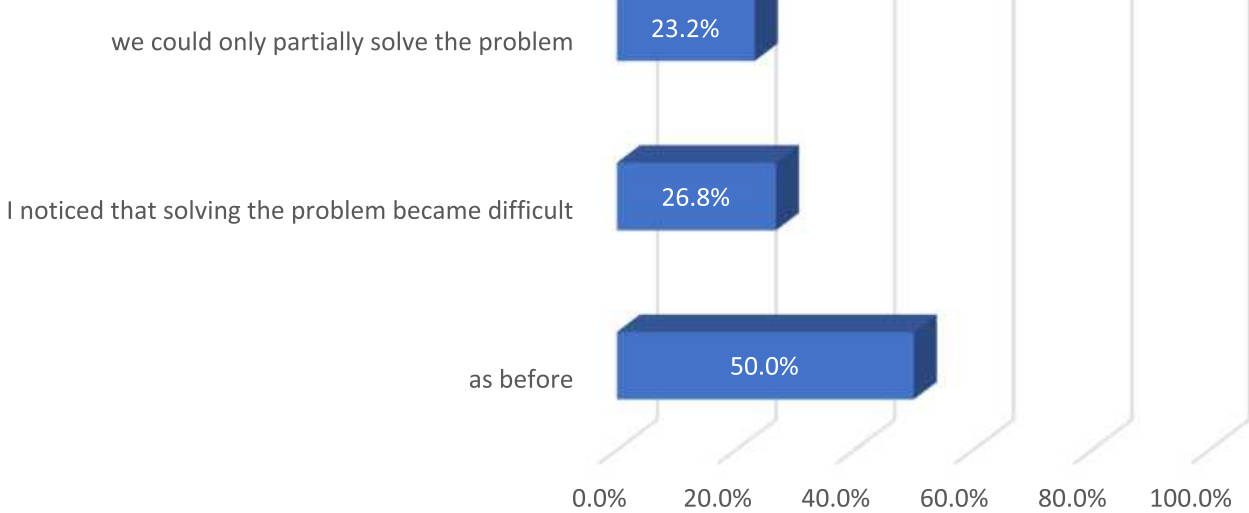

Figure 3 Perception of the remote management of acute diseases during the pandemic.

How did you perceive the remote management of chronic pathology during the pandemic?

were resolved late, by planning to a specialist

inadequate, as many sections were intended for COVID-19 patients only

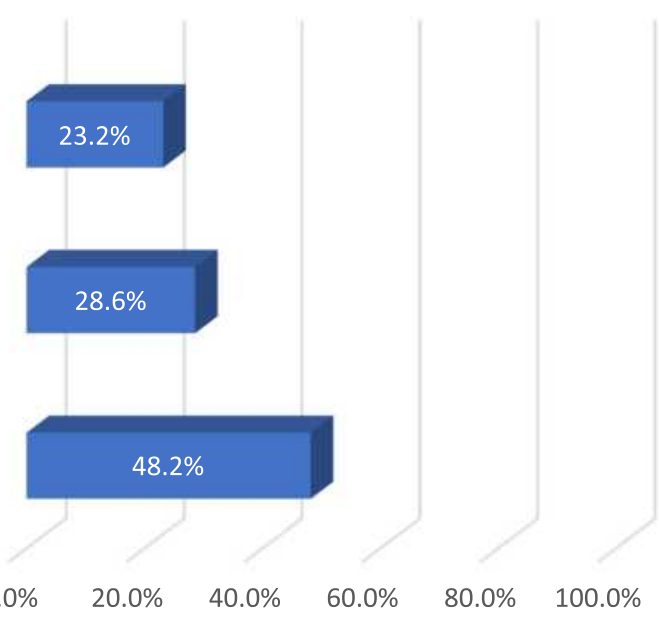

Figure 4 Perception of the remote management of chronic pathology during pandemic.

As regards the general approach of patients through telemedicine tools during the pandemic, family physicians perceived that almost two-thirds of their patients felt as well treated through virtual as in-person visit, a quarter noticed more availability to address health needs through telecare, while less than a tenth of patients experienced a feeling of being neglected (Figure 8.).

The need to continue beyond pandemic the provision of PC through telemedicine was agreed by almost all family doctors and they considered it should be reimbursed continuously as complementary PC service. Only less than a tenth of the physicians surveyed suggested that teleconsultation should not be maintained post-pandemic, given that it does not have the same accuracy as a conventional visit (Figure 9).

\section{Associations Between Perceptions of the Use of Telemedicine During the Pandemic}

As Table 1 shows, family doctors who perceived positive feedback on telemedicine from their patients were more supportive of its further reimbursement and had higher 
How has been affected the surveillance of pregnant women in primary care

during the Covid-19 pandemic?

patients presented to the family doctor's office more often than before, preferring the in person consultation

the frequency of checks at the office during the pandemic did not change

patients have postponed in person visits in GPs' office, preferring teleconsultation with the family doctor

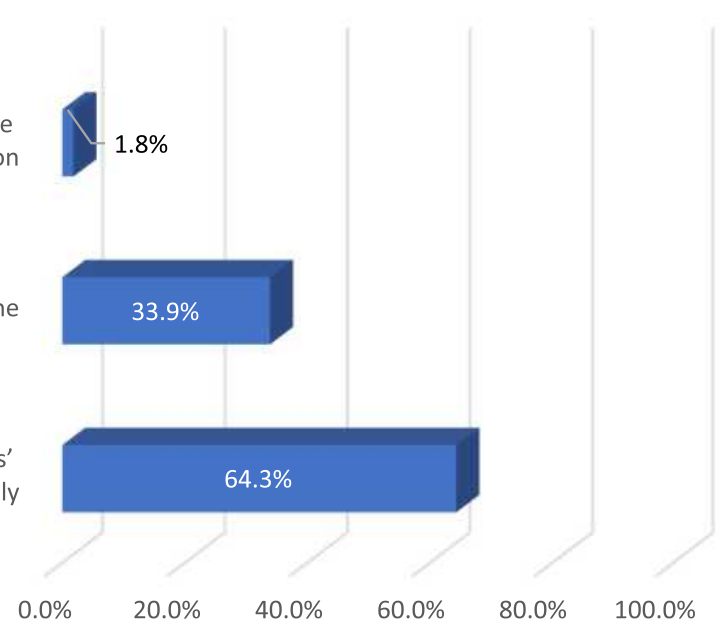

Figure 5 Pregnant women preferences for remote vs in-person consultations perceived by GPs.

How have you noticed that patients react to telemedicine in terms of technology?

they find difficult to use technology and understand it

they were satisfied that they can use technology to simplify solving their healthcare needs (telephone consultations, Whats app video consultation, medical prescription received...

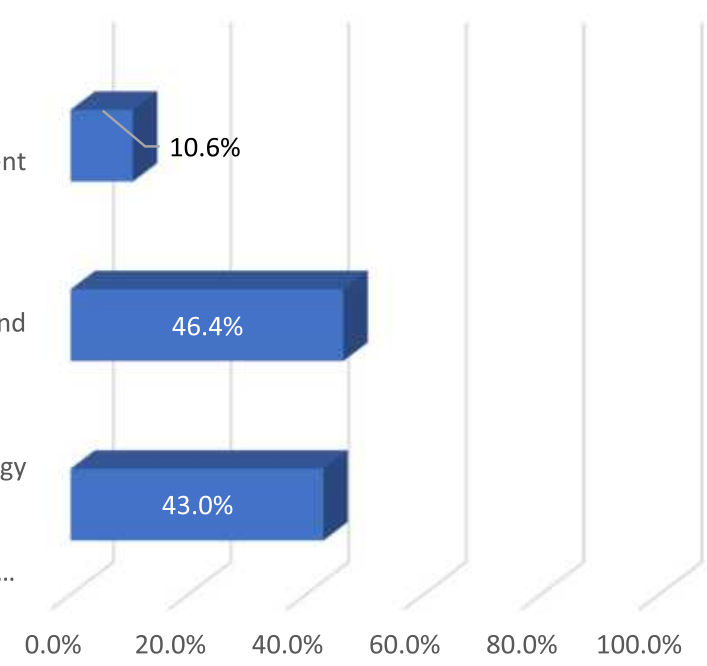

Figure 6 Patients' reaction to telemedicine regarding the use of technology, perceived by GPs.

scores on scales about perception of teleconsultations and reliability in remote decision making and perception of the management of acute and chronic pathology and surveillance of pregnant women in PC during the pandemic (Table 1).

\section{Discussion}

Covid-19 pandemic has made telehealth tech to be in the spotlight. The prompt need constrained physicians to temporarily conduct their usual offline activities by using ICT, gaining new experiences in telecare and affecting their perceptions about tele-decisions. ${ }^{13}$ A Chinese study showed $70 \%$ growth in virtual consultation as compared to conventional visits from March 25th, 2020 until April 17th, 2020. ${ }^{14}$

Physicians are attracted to evidence-based technologies and digital tools that increase patient comfort and adherence, as well as improving and diversifying their clinical practice. ${ }^{15}$ 
How have you noticed that patients react to telemedicine in terms of the quality of healthcare services?

they are indifferent and it seems the same to them

feel insecure about the accuracy of the diagnosis, considering that a classical objective examination is not performed

are equally satisfied with the diagnosis and treatment

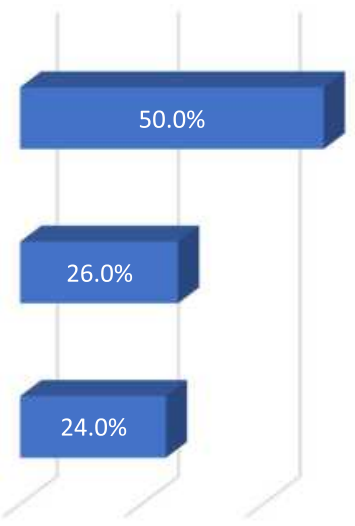

$0.0 \% \quad 20.0 \% \quad 40.0 \% \quad 60.0 \%$

$80.0 \% \quad 100.0 \%$

Figure 7 Patients' reaction to telemedicine regarding the quality of healthcare services provided, as perceived by GPs.

How do you think your patients perceived their family doctor's remote consultation via telemedicine?

the patient felt better and more promptly treated

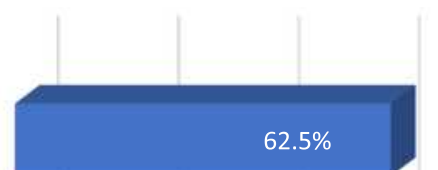

the patient felt equally well treated

the patient felt neglected

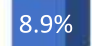

$8.9 \%$

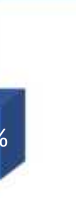

$28.6 \%$

$0.0 \%$

$20.0 \%$

$40.0 \%$

$60.0 \%$

$80.0 \% \quad 100.0 \%$

Figure 8 The general perception of telemedicine by patients according to family doctors.

Unrecognized and not reimbursed before the COVID19 pandemic as a tool for providing PC in Romania, the adoption of teleconsultation made more than half of family physicians perceive it as more difficult and time consuming than in-person visit. Similar to the results of our study on the chronophagous nature of televisits the British GPs reported the time-consuming daily phones, emails and complex electronic medical record protocols. ${ }^{16}$ Unexpectedly, in the United States among physicians currently using telemedicine for consultation, nearly half (48\%) are using it for the first time. ${ }^{17}$

Telehealth services offer benefits to all people registered on the GPs' practice list, increasing access to healthcare services, child development surveillance, ${ }^{18,19}$ remote management of the elderly with chronic comorbidities and helping to prioritize home visits. ${ }^{20}$ Our results are consistent with other studies, revealing the positive perception of physicians and their openness to use telemedicine as an 
Do you think that teleconsultation should be kept as a complementary healthcare service and reimbursed by the Public Health Insurance House $(\mathrm{PHIH})$ after the end of the pandemic?

no, because I do not consider that a telemedicine consultation has the same accuracy as a classic consultation

yes, it should be preserved and reimbursed, together with the in person consultation

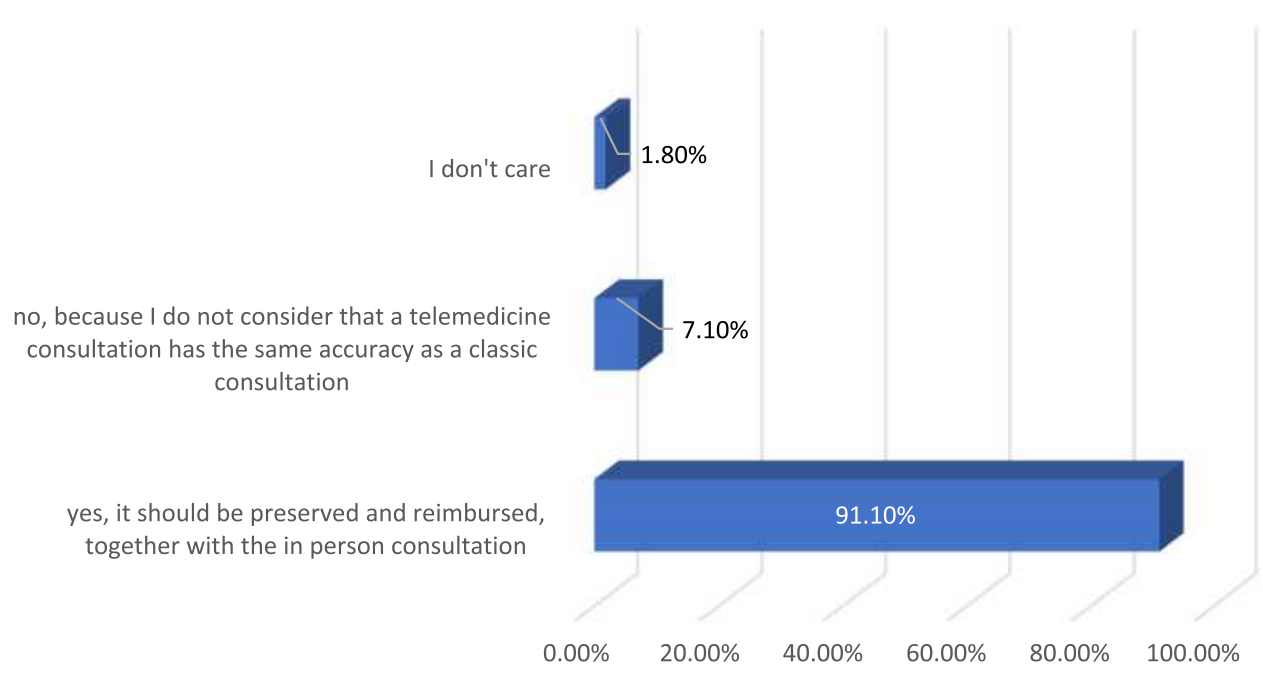

Figure 9 The need to continue post-pandemic the provision of healthcare services through telemedicine in primary care perceived by GPs.

effective way to provide healthcare services during the pandemic. ${ }^{13}$

In addition to the healthcare delivery, there is a considerable utilization of telehealth in medical education. ${ }^{21}$ Some of the family doctors surveyed were

Table I Associations Between Opinions Regarding the Use of Telemedicine

\begin{tabular}{|l|l|l|l|l|}
\hline & I** & 2*** & $3^{* *}$ & $\mathbf{4} * *$ \\
\hline $\begin{array}{l}\text { Perception of teleconsultations and } \\
\text { reliability in remote decision making (I)* }\end{array}$ & - & NS & 0.49 & NS \\
\hline $\begin{array}{l}\text { Perception of the management of acute } \\
\text { and chronic pathology and surveillance } \\
\text { of pregnant women in primary care } \\
\text { during the pandemic (2)* }\end{array}$ & NS & - & 0.33 & NS \\
\hline $\begin{array}{l}\text { Patients' satisfaction with the use of } \\
\text { communication technology to address } \\
\text { their health care needs in primary care, } \\
\text { as perceived by family physicians (3)* }\end{array}$ & 0.49 & 0.33 & - & 0.43 \\
\hline $\begin{array}{l}\text { Opinion of family doctors on the need } \\
\text { for further reimbursement of } \\
\text { telemedicine by the Public Health } \\
\text { Insurance House (PHIH) after the } \\
\text { pandemic (4) }\end{array}$ & NS & NS & 0.43 & NS \\
\hline
\end{tabular}

Notes: * The scale was created by summing the codes of the comprising questions for each participant, as explained in detail in the methodology. ** Only the Pearson correlation coefficients of the statistically significant correlations are described. Abbreviation: NS, non-significant. involved in academic teaching, as assistant professors in the Department of Community Medicine, the discipline of Family Medicine at our university. They expanded their ICT skills and used educational platforms, having previous experience in simulating patients to train students and residents in family medicine. ${ }^{22}$

However, there are limitations to remote medical practice that physicians should be aware of, as the absence of physical examination findings raises uncertainty and concern. ${ }^{23}$ According to a pre-pandemic study, tele-visits led to a correct diagnosis between $65 \%$ and $94 \%$, while standard care protocols were followed in $34 \%$ and $66 \%$ of them. ${ }^{24}$ In the present study, almost two-thirds of GPs (64.3\%) reported uncertainty about the correctness of the diagnosis established using telemedicine and a quarter expressed confidence in making decisions remotely. Surveillance of pregnant women in PC during the COVID-19 pandemic was well supported by telemedicine, as our study showed, with most GPs reporting that registered pregnant women preferred tele-visits. These results correspond to those of another study conducted in two prenatal practices in New York City, where about onethird of the surveillance visits of pregnant women were performed by telehealth (31.8\%). By the fifth week, $56.1 \%$ of family practice visits and $41.5 \%$ of clinic visits were made through telemedicine tools. ${ }^{25}$ Nevertheless, a remote consultation is not intended and will never fully replace an in-person visit, due to the lack of objective clinical signs 
and the interpersonal transmission of non-verbal signals expressing trust and empathy. ${ }^{26}$

Regarding patient satisfaction with teleconsultation as perceived by half of the GPs in our study, this is consistent with other studies. A study pointed out that $95 \%$ of patients who were treated via telemedicine during the COVID-19 pandemic rated it to be useful as in-person visit. ${ }^{27}$ Patients identified the convenience, efficiency, communication, confidentiality, and comfort of their own supportive environment as important areas to consider when evaluating virtual PC consultations versus office visits. ${ }^{28}$ Other studies have shown that in countries where remote healthcare services were used pre-pandemic, $86 \%$ of patients were satisfied with the virtual interaction. In addition, the acceptance of teleconsultation seemed to be linked to patients' trust with their local health system and staff. ${ }^{29}$ A survey of patients' perceptions of telehealth in the United States of America during the COVID-19 pandemic found satisfaction was high both in new and previous users of telehealth but new users were more motivated to avoid waiting rooms and potential infection. ${ }^{30}$

Yet, many patients have difficulties in understanding and using ICT. As our study showed, the technological literacy of patients varies, and some of them, $46.5 \%$ encountered barriers to connection through telemedicine due to lack of access to technology and insufficient social assistance. People with poor technological literacy, who lack access to the Internet, need help from volunteers and technology companies who can create low-cost plug-andplay telemedicine devices. ${ }^{31}$

Despite the fact that there remains an amount of skepticism and uncertainty regarding telemedicine, especially regarding the efficiency, safety and adequacy of existing regulations, there are studies that recommend and support the continuity of telehealth activities beyond the COVID-19 pandemic. ${ }^{13}$ In our study, most family physicians $(91.1 \%)$ considered necessary to continue PC provision through telemedicine post-pandemic. In Brazil, it has been signaled that the lack of regulation on the use of telemedicine was an important barrier to assist patients and $64.39 \%$ of the physicians wanted a regulation that would allow the expansion of the telehealth care services. ${ }^{32}$ Surprisingly, another study showed that when the pandemic ends, only one-fifth of physicians using telemedicine tools expect to use them significantly more than before the pandemic. ${ }^{33}$

A literature review analyzing the PC provision in 6 countries suggests that COVID-19 is testing healthcare systems, even in well-resourced countries. ${ }^{34}$ As our study has shown, government policies play a significant role in managing this difficult period of long-term COVID-19 pandemic. The decision of the Romanian government to adopt and reimburse teleconsultations during the pandemic and to continue today, has benefited patients and protected the health of medical staff.

Having yet no definitive endpoint, this pandemic requires creative solutions, such as the scale-up of telehealth, contributing to decrease its impact. ${ }^{21}$ New telehealth solutions have emerged, improving the quality of tele-decisions. ${ }^{35}$ The latest inventions used to combat the novel SARS-CoV-2 infection are artificially intelligent (AI)-based conversational agents, known as health chatbots. They enable patients to interact with software applications that use AI-based tools, accessed through a website or social media messaging platforms. ${ }^{36-39}$

Before the pandemic, a PC chatbot system was created to assist GPs by automating the patient intake process. This interactive system called Mandy is not designed as a diagnostic or clinical decision-making tool but an assistant, helping to free up the time of family doctors for more meaningful interactions with patients. ${ }^{40}$ These technologies are multi-tasking: ask and answer questions, create health records, complete forms and generate reports.

During the pandemic, the WHO Technology program developed a chatbot to fight COVID-19, an initiative which can be accessed via WhatsApp and Facebook messenger. It is a new WHO interactive chatbot that aims to combat COVID19 misinformation. ${ }^{41}$ The use of health chatbots to combat COVID-19 is a practice still in its infancy. Further research will lead better understanding of this novel technology's applications and improve their functionalities and usefulness. ${ }^{39}$

The speed with which telehealth is progressing can have a significant effect on advancing healthcare in the future. One should view the current crisis as both a challenge and an opportunity to assess the impact of digital tools on access to care, quality of care, and the financial impact on the healthcare system. ${ }^{15}$

\section{Implications for Practice}

The COVID-19 pandemic continues and patients with acute and chronic conditions should be cared for safely. Telemedicine has existed for decades, but its widespread adoption in this long-term crisis has integrated it into the daily routine, increasing access to care and helping GPs to diversify the provision of PC services. As our study showed, family doctors considered that telemedicine should be implemented not only as a temporary alternative during the crisis, but beyond the pandemic. Telemedicine-based training for family 
physicians can improve capacity building and streamline office management.

\section{Implications for Health Policy}

Although telemedicine was initially reimbursed only during the state of emergency and alert in Romania, its contribution to pandemic mitigation and positive perception by both patients and healthcare providers influenced health policy and led PHIH to continue its reimbursement.

\section{Conclusion, Limitations, and Future Works}

This study showed the positive perception of family doctors who quickly adapted to teleconsultations, despite concerns about decision-making remotely. The recognition and reimbursement of telemedicine for the first time by PHIH have shed light on the shadows of the pandemic. The time-consuming nature of teleconsultations, the uncertainty in tele-decisions and difficulties of some patients in using communication technology were seen as shadows of the use of telemedicine. However, most of the GPs surveyed agreed with the need for its further reimbursement.

Research has limitations, as does our study. First, data on rural/urban areas, gender and age of participants are missing. In addition to data protection considerations, the questionnaire was developed in the emergency state of the COVID-19 pandemic and focused on the GPs' perception of the use of telemedicine, considered at that time as a temporary alternative during the crisis. Second, the limit of having only a response rate of $51 \%$ is explained by the demanding period, in a country facing an acute shortage of health professionals and a lack of protective equipment, which has all distracted the doctors in our survey. Third, asking physicians to comment on patients' perceptions of the rapid transition from traditional to virtual visit is another limitation. As GPs are selected by people who enroll and want to remain on the list of practice for a long time, knowing their satisfaction with this new critical approach to healthcare is in the interest of both parties.

Future work should focus on creative and innovative solutions that integrate telemedicine as a complementary form of PHS delivery and streamline the management of general practice, not only as a temporary response to a crisis, but as a proactive method to increase access to quality care. Studies on the need for telemedicine-based training for family physicians can improve capacity building and research on patients' perceptions of virtual care can help to build trust and satisfaction.

\section{Abbreviations}

COVID,19, Coronavirus disease 2019; GPs, general practitioners; PHIH, Public Health Insurance House; PHA, public health authorities; PC, primary care; WHO, World Health Organization; ICT, information and communications technologies; AI, artificial intelligence.

\section{Ethics Approval and Informed Consent}

This study received the approval of the Ethics Committee of the College of Physicians from Cluj-Napoca, Romania, Nr. 984, April 7, 2020. The guidelines outlined in the Declaration of Helsinki were followed.

\section{Acknowledgments}

Special thanks to the family doctors that have responded to our online survey.

\section{Author Contributions}

All authors made a significant contribution to the work reported, whether that is in the conception, study design, execution, acquisition of data, analysis and interpretation, or in all these areas; took part in drafting, revising or critically reviewing the article; gave final approval of the version to be published; have agreed on the journal to which the article has been submitted; and agree to be accountable for all aspects of the work.

\section{Funding}

This research received no external funding.

\section{Disclosure}

The authors declare no conflicts of interest in this work.

\section{References}

1. Keesara S, Jonas A, Schulman K Covid-19 and health care's digital revolution. $N$ Engl J Med, 2020 382: e82.

2. Kichloo A, Albosta M, Dettloff K, et al. Telemedicine, the current COVID-19 pandemic and the future: a narrative review and perspectives moving forward in the USA. Fam Med Community Health. 2020 8(3):e000530. doi:10.1136/fmch-2020-000530

3. Sood S, Mbarika V, Jugoo S, et al. What is telemedicine? A collection of 104 peer-reviewed perspectives and theoretical underpinnings. Telemed J E Health 2007; 13:573-590. doi:10.1089/tmj.2006.0073.

4. Oh H, Rizo C, Enkin M, Jadad A What is ehealth (3): a systematic review of published definitions. $J$ Med Internet Res 2005; 7: e1. doi:10.2196/jmir.7.1.e1.

5. Bokolo A Jnr. Integrating telemedicine to support digital health care for the management of COVID-19 pandemic. Int $J$ Healthcare Manage, 2021. doi:10.1080/20479700.2020.1870354. 
6. Dos Santos Puga ME, de Assis Reis FS, Milby KM, et al. Telehealth interventions in the context of the COVID-19 pandemic: protocol for a scoping review. 2020. doi:10.1590/SciELOPreprints.353.

7. Wootton R, Geissbuhler A, Jethwani K et al. Long-running telemedicine networks delivering humanitarian services: experience, performance and scientific output. Bull World Health Organ 2012; 90:341347D. doi:10.2471/BLT.11.099143.

8. WHO Global Observatory for eHealth, 2010 Telemedicine: Opportunities and Developments in Member States: Report on the Second Global Survey on eHealth. World Health Organization. Available from: http://whqlibdoc.who.int/publications/2010/ 9789241564144_eng.pdf. Accessed 25 October 2020.

9. Bokolo AJ Exploring the adoption of telemedicine and virtual software for care of outpatients during and after COVID-19 pandemic. Ir J Med Sci. 2021;190(1):1-10. doi:10.1007/s11845-020-02299-z

10. Bokolo A Jnr. Use of telemedicine and virtual care for remote treatment in response to COVID-19 pandemic. J Med Syst. 2020;44 (7):132. doi:10.1007/s10916-020-01596-5

11. Ranganathan $\mathrm{C}$, Balaji S Key factors affecting the adoption of telemedicine by ambulatory clinics: insights from a statewide survey. Telemed J E Health. 2020;26(2):218-225. doi:10.1089/ tmj.2018.0114.

12. World Health Organization. 2020. Strengthening the health systems response to COVID-19. Available from http://www.euro.who.int/ data/assets/pdf_file/0007/436354/strengthening-health-systemsresponse-COVID-19-technical-guidance-1.pdf. Accessed October 26, 2020.

13. Helou S, El helou E, Abou-Khalil V, et al. The effect of the covid-19 pandemic on physicians' use and perception of telehealth: the case of Lebanon. Int J Environ Res Public Health. 2020 17(13):4866. doi:10.3390/ijerph17134866

14. Song X, Liu X, Wang C (2020) The role of telemedicine during the COVID-19 epidemic in China - experience from Shandong province. Crit Care 24(1):178.doi:10.1186/s13054-020-02884-9.

15. Hodgkins M, Barron M, Jevaji S et al. Physician requirements for adoption of telehealth following the SARS-CoV-2 pandemic. $N p j$ Digit Med. 2021,4,19. doi:10.1038/s41746-021-00390-y.

16. Thornton J Covid-19: how coronavirus will change the face of general practice forever. BMJ 2020;368:m1279. doi:10.1136/bmj.m1279.

17. Abdel-Wahab M, Rosenblatt E, Prajogi B, Zubizarretta E, Mikhail M Opportunities in telemedicine, lessons learned after COVID-19 and the way into the future. Int J Radiat Oncol Biol Phys. 2020;108 (2):438-443. doi:10.1016/j.ijrobp.2020.07.006.

18. Valea A, Silaghi A, Ghervan C, et al. Morbid child obesity with possible Rohhadnet-Rohhad syndrome. Acta Endocrinologica (Buc). 2014, X, 3: 515-524.

19. Lotrean L, Popa M, Santillan EA, Florea M. Methodological challenges in research regarding the lifestyle of school children. Revista De Cercetare Si Interventie Sociala. 2014, 44, 321-331

20. Florea M, Puia A, Pop RS.The family as recipient and provider of home care: a primary care perspective, In: "Palliative Care", editors John Stones M, Cascella M. 2020. Available from: https://www. intechopen.com/online-first/the-family-as-recipient-and-provider-ofhome-care-a-primary-care-perspective.

21. Doraiswamy S, Abraham A, Mamtani R, Cheema S Use of telehealth during the COVID-19 pandemic: scoping review. J Med Internet Res 2020;22(12):e24087. doi:10.2196/24087

22. Florea M, Țalu Ş, Țalu M. Patient simulation experience in family medicine residents' education. In: IFMBE Proceedings MEDITECH 2009 "International Conference on Advancements of Medicine and Health Care through Technology", 23-26 Sept 2009, Cluj-Napoca, Romania: Springer-Verlag GmbH, Heidelberg, Germany. 26, 25-28. doi:10.1007/978-3-642-04292-8_6.

23. Iyengar K, Jain VK, Vaishya R Pitfalls in telemedicine consultations in the era of COVID 19 and how to avoid them. Diabetes Metab Syndr. 2020;14 (5):797-799.
24. Schoenfeld AJ, Davies JM, Marafino BJ, et al. Variation in quality of urgent health care provided during commercial virtual visits. JAMA Intern Med. 2016;176 (5):635-642. doi:10.1001/jamainternmed.2015.8248.

25. Madden N, Emeruwa UN, Friedman AM, et al. Telehealth uptake into prenatal care and provider attitudes during the COVID-19 pandemic in New York City: a quantitative and qualitative analysis. Am J Perinatol. 2020;37(10):1005-1014. doi:10.1055/s-0040-1712939.

26. Khanom M, Chowdhury S Telehealth consultation in the COVID-19 era: utilisation and challenges of virtual platform. Chattogram MaaO-Shishu Hosp Med Coll J, 202019 (2): 3-4. doi:10.3329/cmoshmcj. v19i2.50013www.banglajol.info/index.php/CMOSHMCJ.

27. Wosik J, Fudim M, Cameron B, et al. Telehealth transformation: COVID-19 and the rise of virtual care. J Am Med Inform Assoc. 2020; 27:957-962. doi:10.1093/jamia/ocaa067.

28. Powell RE, Henstenburg JM, Cooper G, et al. Patient perceptions of telehealth primary care video visits. Ann Fam Med 2017; 15: 2259.

29. Sabesan S, Kelly J, Evans R, Sarah Larkins S A tele-oncology model replacing face-to-face specialist cancer care: perspectives of patients in North Queensland. J Telemed Telecare. 2014, 20(4) 207-211. doi:10.1177/1357633X145292.

30. Holtz BE Patients perceptions of telemedicine visits before and after the coronavirus disease 2019 pandemic. Telemed J E Health. 2020;01:01. doi:10.1089/tmj.2020.0168.

31. Calton B, Abedini N, Fratkin M Telemedicine in the time of coronavirus. J Pain Symptom Manage. 2020;60(1):e12-e14. doi:10.1016/j.jpainsymman.2020.03.019

32. Caetano R, Silva AB, Guedes ACCM, et al. Challenges and opportunities for telehealth during the COVID-19 pandemic: ideas on spaces and initiatives in the Brazilian context. Cadernos De Saúde Pública. 2020 36(5),e00088920.

33. Sermo. COVID-19 HCP Sentiment Surveys.2020. Part 1: physician engagement with patients and remote/telehealth experiences. Available from:. https://www.sermo.com/hcp-sentiment-study-series/. Accessed June 24, 2020.

34. Huston P, Campbell J, Russell G, et al. COVID-19 and primary care in six countries. BJGP Open. 2020;4(4): bjgpopen20X101128. doi:10.3399/bjgpopen20X101128.

35. Deldar K, Tara F, Bahaadinbeigy K, Khajedaluee M, Tara M, Data amodel for teleconsultation in managing high-risk pregnancies: design and preliminary evaluation. JMIR Med Inform. 2017;5(4): e52. doi:10.2196/medinform.8393.

36. Battineni G, Chintalapudi N, Amenta F AI chatbot design during an epidemic like the novel coronavirus. Healthcare MDPI AG;2020;8 (2): 154. doi:10.3390/healthcare8020154.

37. Rodsawang $\mathrm{C}$, Thongkliang $\mathrm{P}$, Intawong $\mathrm{T}$, Sonong A, ThitiwatThana Y, Chottanapund S. Designing a competent chatbot to counter the covid-19 pandemic and empower risk communication in an emergency response system. OSIR J. 2020; 30: :2.

38. Walwema J. The WHO health alert: communicating a global pandemic with WhatsApp. J Business Techn Commun. 2020. doi:10.1177/1050651920958507.

39. Almalki M, Azeez F. Health Chatbots for fighting COVID-19: a scoping review. ACTA INFORM MED 2020, 28(4): 241-247. doi:10.5455/ aim.2020.28.241-247

40. Ni L, Lu C, Liu N, Liu J. MANDY: towards a smart primary care chatbot application. In: Chen J, Theeramunkong T, Supnithi T, Tang X (eds) Knowledge and Systems Sciences. KSS. Communications in Computer and Information Science. 2017, 780. Springer, Singapore. doi:10.1007/978-981-10-6989-5_4.

41. WHO. 2020. WHO launches a chatbot on Facebook Messenger to combat COVID-19 misinformation. Available from: https://www. who.int/news-room/feature-stories/detail/who-launches-a-chatbotpowered-facebook-messenger-to-combat-covid-19-misinformation. Acssessed February 23, 2021. 


\section{Publish your work in this journal}

The International Journal of General Medicine is an international, peer-reviewed open-access journal that focuses on general and internal medicine, pathogenesis, epidemiology, diagnosis, monitoring and treatment protocols. The journal is characterized by the rapid reporting of reviews, original research and clinical studies

across all disease areas. The manuscript management system is completely online and includes a very quick and fair peer-review system, which is all easy to use. Visit http://www.dovepress.com/ testimonials.php to read real quotes from published authors. 